\title{
In-House Implications of a 1-Year Retrospective Analysis of the Psychiatric Consultation-Service in a German University Hospital
}

\author{
Maximilian Gahr, Markus Schmid, Roland W. Freudenmann, Carlos Schönfeldt-Lecuona \\ Department of Psychiatry and Psychotherapy III, Ulm University, Ulm, Germany \\ E-mail: \{maximilian.gahr, markus.schmid, roland.freudenmann, carlos.schoenfeldt\}@uni-ulm.de \\ Received February 11, 2011; revised March 12, 2011; accepted March 14, 2011
}

\begin{abstract}
Objective: Due to major differences in patient populations, consultants and hospital settings of single-centres structured analyses of the psychiatric consultation-service (PCS) might be considered as an internal statistical appraisal for quality and efficacy improvements of the focused PCS. Method: The patient population given by the original documentation schedules of psychiatric consultations performed in the University hospital of Ulm in the year 2008 were analysed for the following variables: sex, age, requesting department, based problem or consultation query, allocated psychiatric diagnosis, therapeutic suggestion, occurrence and number of psychiatric consultations within the same case, acuteness level, diagnosis according to ICD-10 F and therapeutic recommendation. Results: In a total of $n=656$ consultations most frequent questions were for: "medication", "diagnostic suggestions" and "no specific question”, whereupon "no question” was given in $19 \%$. A statement concerning the urgency-level of the consultation was present in $10.5 \%$. Organic mental disorders, mood disorders, neurotic, stress-related and somatoform disorders formed $72 \%$ of all diagnoses. Pharmacotherapy was most frequently recommended. The detected shortcomings were mostly the result of heterogenous documentation. Conclusions: Frequent absence of relevant information throughout the consultation process gives reason for interdisciplinary arrangements to create a rational algorithm for PCS. Based on our findings standardization of our consultation reports was established by means of a standard form and a pocket-booklet which provides diagnostic guidelines and therapeutic recommendations.
\end{abstract}

Keywords: Consultation- and Liaison-Psychiatry, Standardization, Quality Control

\section{Introduction}

Mental and behavioural disorders have been reported to be present among approximately one third of general hospital inpatients [1-4]. Therefore, PCS plays a preponderant role in the multidisciplinary treatment approach when dealing with multimorbid patients, which suffer secondarily psychiatric symptoms as a consequence of a somatic disease or have a genuine comorbid psychiatric disorder. An improvement in diagnostic and therapeutic accuracy of comorbid psychiatric disorders would be desirable since psychiatric comorbidity has been described to be associated with poor clinical outcome, extended hospitalization and increased mortality in medically ill patients [5-8]. A small number of studies have been conducted in the last decades to picture the PCS in single centres [7,9-12]. As a result of major dif- ferences in patient populations, consultants and hospital settings the findings rendered by these studies are heterogeneous and primarily only valid for the particular institution. In this light, single-centre data are not generalisable and not appropriate to make comparisons among other centres in order to retrieve general insights in consultation psychiatry. Structured analyses of the PCS might primarily be considered as an internal appraisal that offers the statistical foundation for quality and efficacy improvement of the focused consultation-service.

Considering that clinical outcome and hospitalization time in medically ill patients with psychiatric comorbidity is deeply related with the accomplishment of the consultation-liaison psychiatry service, we aimed to assess the performance of our department. With the ultimate aim of optimizing our PCS in the matter of adjusted standardization and quality control a retrospective cross- 
sectional analysis of all psychiatric consultations at the University Hospital Ulm (Ulm, Germany) was carried out observing a 1-year period. Therefore, an in-house inventory was developed to gain data (see methods) from the documentation schedules for further analysis. Based on shortcomings in documentation, that should be identified in our analysis, we first intended to create a standard form with particular items according to detected idiosyncracies that should replace the former blank documentation schedules. In addition, composing a pocket-booklet with useful information for the consulting psychiatrist was proposed. This pocket-booklet should contain information regarding diagnostic criteria, differential diagnosis and treatment guidelines of the most frequent diseases within our PCR to render an improved and more homogenous outcome possible.

\section{Materials and Methods}

Data used for further analysis was obtained from the original documentation schedules of psychiatric consultations performed by physicians belonging to the Department of Psychiatry and Psychotherapy III of the University hospital Ulm. We collected data from all psychiatric consultations of the year 2008 (between the $1^{\text {st }}$ of January and the $31^{\text {th }}$ of December) performed by our consultants in other medical departments of the University Hospital in Ulm. Selected data (patient and case related data) from these documents was retrieved and assembled in a separate chart review assessing different issues (see below). For data analysis two perspectives were regarded: 1) The information standing on the request schedule (requesting department) and 2) the psychiatric evaluation and recommendation documented.

\subsection{Data Assessment from the Request Schedule}

Following aspects were assessed from the request schedule: "Gender" and "age” of the patient, "requesting department”, "based problem or consultation query”, “allocated psychiatric diagnosis”, “therapeutic suggestion”, "occurrence and number of psychiatric consultations within the same case", and existence of a statement indicating the "acuteness level" and accordingly the "number of affirmated emergency consultations". In this context, documentation schedules were scrutinized for the existence of explicit declarations of urgency, respective emergency and whether this indication (e.g. emergency: yes/no) could be confirmed by the consulted psychiatrist of our department. In order to identify and accurately quantify the most frequently formulated questions by the colleagues of other disciplines requesting a psychiatric consultation in the documentation schedules we decided to build subcategories. Those were created having regard to frequently articulated problems that were standing out within a first view of the data and in consideration of our clinical experience. Here we focused on explicit verbal formulations that displayed distinct questions or that were denoted with a question mark. Recurring reasons for a psychiatric consultation were as followed: "no question documented”, “medication procedure?”, “diagnostic procedure?”, “diagnosis?”, “suicidal tendencies or risk?”, “forensic reason?” (in a broader sense, e.g. patient's capacity to give informed consent to a medical procedure or the necessity of installation a legal representative), "indication for transferring the patient to the psychiatry department?" and questions that contained combinations of the above explained subcategories: "diagnostic and medication procedure", "forensic reason and therapy procedure”, "forensic reason and diagnosis”.

\subsection{Data Assessment from the Psychiatric Evaluation and Recommendation}

Following issues were assessed from the psychiatric evaluation and recommendations: "Diagnoses"; those were registered according to international classification of disorders (ICD-10), chapter F from the world health organization [13]. Therapeutic recommendations documented were also distributed to a set of subcategories: “no specific recommendation”, "medication”, “indication for transferring the patient to the psychiatric department", "forensic reason”, “diagnostic procedure (e.g. MRI scan of the brain, cerebrospinal fluid)", "medication and psychotherapy”, “psychotherapy”.

\section{Results}

Between the $1^{\text {st }}$ of January and the $31^{\text {th }}$ of December 2008 a total of 656 psychiatric consultations were performed by our department.

1) Assessed Data gained from the request schedule, independent of the psychiatric evaluation and recommendation, showed following results:

Sex and age: Fifty nine percent of the patients were female $(n=390)$ and $41 \%(n=266)$ were male. Regarding the different age-groups, $2 \%$ of the patients $(n=15)$ were older than 90 years, $14 \%(n=90)$ showed an age between 80 and $89,16 \%(\mathrm{n}=107)$ between 70 and 79 , $18 \%(n=119)$ between 60 and 69, 14\% $(n=94)$ between 50 and 59, 15\% $(n=99)$ between 40 and 49, 10\% $(n=66)$ between 30 and 39, 10\% $(n=63)$ between 20 and 29 and $1 \%(n=3)$ were younger than 20 years (see Figure 1$)$. More than half of the patients $(50.5 \%, \mathrm{n}=331$ ) were 60 years or older on time of individual consultation. 
Requesting department: Considering the requesting department, $79 \%$ of all consultations were performed in four departments: internal medicine $(n=230 / 35 \%)$, surgery $(n=173 / 26 \%)$, geriatrics $(n=64 / 10 \%)$ and neurology ( $\mathrm{n}=54$ / 8\%). Details can be seen in Table 1 .

Consultation query: The majority of questions stated on the request schedule $(84 \%, \mathrm{n}=552)$ could be allocated to four subcategories: "medication" $(27 \%, n=180)$, "diagnostic suggestions" (20\%, $n=129)$, "no specific question" (19\%, $\mathrm{n}=124)$ and "suicidal tendency or risk" $(18 \%, n=119)$. For details see Table 2.

2) Assessed Data gained from the documented psychiatric evaluation and recommendation, showed following results:

Diagnostic codes: Three ICD-10 F diagnostic codegroups covered $72 \%$ of all diagnoses: Organic, including symptomatic, mental disorders (ICD-10 F00-F09) were n = $193(30 \%)$, mood (affective) disorders (ICD-10 F30F39) had $n=192$ (29\%), neurotic, stress-related and somatoform disorders (ICD-10 F40-F48) $n=88$ (13\%). No psychiatric diagnosis was given in $12 \%$ of the cases $(\mathrm{n}=78)$. Details are listed in Table 2.

Therapeutic recommendations: Pharmacotherapy was the therapeutic recommendation in 59\% $(\mathrm{n}=384)$, whereas a non classifiable recommendation respectively no specific psychiatric recommendation was articulated in $16 \%(n=103)$. Transfer to the department of psychiatry for continuative inpatient treatment (independent of diagnosis) was recommended in $7 \%(n=44)$.

Forensic recommendation: In $6 \%(n=40)$ of all consultations a forensic recommendation in the broader sense was documented (e.g. a statement concerning patient's capacity to give informed consent to a medical procedure or the necessity of installation a legal representative). Details can be seen in Table 3.

Consultation frequency: Regarding the consultation frequency per case, $7 \%$ of all patients $(n=48)$ have been consulted twice, whereas $2 \%(\mathrm{n}=14)$ received a psychiatric consultation three times, and $1 \%(n=7)$ had four psychiatric consultations. In only $0.5 \%$ of the cases $(n=$ 3) had to come across the psychiatrist more than four times.

Emergency consultation: Consultation request texts were also analysed for the existence of specific declarations according to the urgency of the psychiatric consultation, respectively to a possible emergency situation. Existing designations of this kind were inspected whether the psychiatrist had acknowledged the particular labelling or not. A statement indicating the priority of the inquired consultation as urgent or emergency was found in $n=69$ (10.5\%) with $n=40$ (6\%) of declared and subsequent acknowledged emergencies and $n=29$ (4.5\%) of so declared but later on not confirmed emergencies.
Table 1. Requesting departement in a total of $n=656$ consultations [female $n=390(59 \%)$; male $n=266(41 \%)$ ].

\begin{tabular}{ll}
\hline Requesting departement & \multicolumn{1}{c}{$\mathrm{n} / \%$} \\
\hline Internal medicine & 230 / 35\% \\
Surgery & 173 / 26\% \\
Geriatrics & $64 / 10 \%$ \\
Neurology & 54 / $8 \%$ \\
Otorhinolaryngology & 26 / 4\% \\
Gynecology & 22 / 3\% \\
Urology & 19 / 3\% \\
Dermatology & 14 / $\%$ \\
Anesthesia & 14 / 2\% \\
Orthopedics & 13 / 2\% \\
Nuclear medicine & 11 / 2\% \\
Neurosurgery & 8 / 1\% \\
Ophtalmology & $6 / 1 \%$ \\
Child-and adolescent psychiatry & 2 / 1\% \\
\hline
\end{tabular}

Table 2. Question categories and psychiatric diagnoses.

\begin{tabular}{|c|c|c|c|}
\hline Question category: & $\mathrm{n} / \%$ & $\begin{array}{c}\text { Diagnosis } \\
(\mathrm{ICD}-10 \mathrm{~F}) \text { : }\end{array}$ & $\mathrm{n} / \%$ \\
\hline Medication & $180 / 27 \%$ & ICD-10 F 0 & $193 / 30 \%$ \\
\hline Diagnostic & 129 / 20\% & ICD-10 F 1 & 37 / 6\% \\
\hline None & $124 / 19 \%$ & ICD-10 F 2 & 35 / 5\% \\
\hline Suicidal tendency & $119 / 18 \%$ & ICD-10 F 3 & 192 / 29\% \\
\hline Forensic & $47 / 7 \%$ & ICD-10 F 4 & 88 / 13\% \\
\hline $\begin{array}{l}\text { Diagnostic + } \\
\text { medication }\end{array}$ & 33 / 5\% & ICD-10 F 5 & $4 / 1 \%$ \\
\hline Transfer to psychiatry & $18 / 3 \%$ & ICD-10 F 6 & $28 / 4 \%$ \\
\hline Forensic and therapy & $3 / 0.5 \%$ & ICD-10 F 7 & $0 / 0 \%$ \\
\hline \multirow[t]{3}{*}{ Forensic and diagnosis } & $3 / 0.5 \%$ & ICD-10 F 8 & $1 / 0 \%$ \\
\hline & & ICD-10 F 9 & $0 / 0 \%$ \\
\hline & & $\begin{array}{l}\text { No psychi- } \\
\text { atric diag- } \\
\text { nosis }\end{array}$ & $78 / 12 \%$ \\
\hline
\end{tabular}

Table 3. Therapeutical recommendations.

\begin{tabular}{lc}
\hline $\begin{array}{l}\text { Category of therapeutical } \\
\text { recommendation }\end{array}$ & $\mathrm{n} / \%$ \\
\hline Medication & 384 / 59\% \\
No specific & $103 / 16 \%$ \\
Transfer to psychiatry & $44 / 7 \%$ \\
Forensic & $40 / 6 \%$ \\
Further diagnostic procedures & 37 / 5\% \\
Medication and psychotherapy & 33 / 5\% \\
Psychotherapy & $15 / 2 \%$ \\
\hline
\end{tabular}

Further diagnostical procedures, recommended in addition to any of the above mentioned recommendations at $\mathrm{n}=84(13 \%)$ consultations; Additional recommendation with psychosocial or forensic character at $\mathrm{n}=38$ (6\%).

\section{Discussion}

Several studies have reported an association between psychiatric comorbidity and poor clinical outcome, extended hospitalization and increased mortality in medically ill patients. Thus, improvement in diagnostic and 


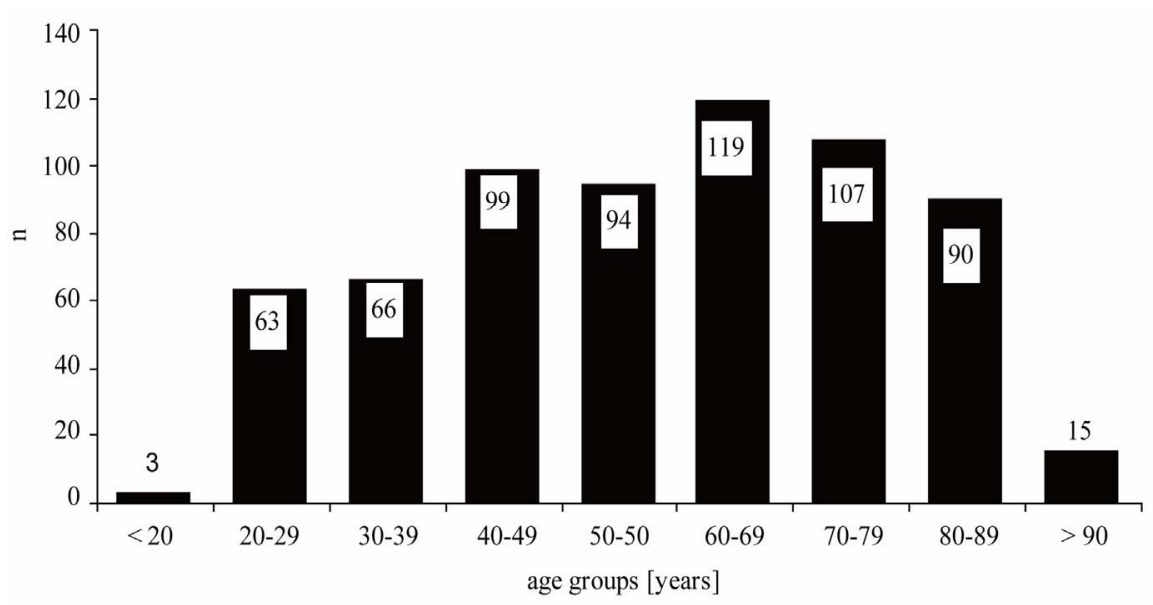

Figure 1. Age structure of the total population on time of individual consultation $(n=656)$.

therapeutic accuracy of comorbid psychiatric disorders would be essential to ameliorate the actual scenery. Enhancing the expertise of PCS is the key issue of being more efficient in the treatment of multimorbid patients that present psychiatric co-morbidity. The accurate analysis of our work has to be conceived as quality control, and should be used to better characterize the needs of system implementation.

Regarding our data, the department of internal medicine accounted for the majority of psychiatric consultations (35\%), followed by the surgery department (26\%); this is in line with other published studies [9,14-17]. The analysis of the consultation query showed that the majority of questions documented on the request schedule could be allocated to four subcategories ranging from "pharmacotherapy" (27\%), followed by "diagnostic suggestions” (20\%), "no specific question” (19\%), and "suicidal tendency or risk" in $18 \%$ of the cases. On the other hand, the analysis of the data gained from the documented psychiatric evaluation and recommendation showed that organic mental disorders (including symptomatic and substance induced disorders ICD-10 F00F09) were the most frequently diagnosed (30\%), followed by mood (ICD-10 F30-F39 = 29\%) and neurotic, stress-related and somatoform disorders (ICD-10 F40F48 $=13 \%$ ). Pharmacotherapy was the most frequently therapeutic recommendation in 59\%, similarly to previously reported investigations $[10,14,17]$. Transfer to the department of psychiatry for continuative inpatient treatment (independent of diagnosis) was recommended only in $7 \%$ of the consultations. Forensic recommendations as are e.g. a statement concerning patient's capacity to give informed consent to a medical procedure or the necessity of installation of a legal representative, was in $6 \%$ of all consultations. $7 \%$ of all patients received a psychiatric consultation twice; whereas $2 \%$ received it three times (1.5\% had four psychiatric consultations).
$10.5 \%$ of requested consultations were declared as emergency consultations by the requesting department; in only the half of them the consultation could be confirmed as emergency.

All in all, the shortcomings of our PCS that were detected in our analysis seemed to be the consequence of lacking standardization. The request schedule plays a very important role when considering the efficiency of the PCS, since the psychiatric consultant has to orient his work to the question stated on the requesting schedule. A consultation schedule without precise question ("no specific question" in our study was provided $19 \%$ of the cases) could trigger at least two extreme positions: the consultant rejects the evaluation and demands a new schedule or he has to evaluate all anamnestical, medical and psychopathological aspects of the case. A third possibility is contacting the requesting department and trying to find out the reason for the consultation; all three positions resulting in an unfeasible waist of resources and time. In consideration of unspecific or even missing consultation questions an interdisciplinary arrangement to create a rational algorithm for reasonable processing of psychiatric consultation seems reasonable from a quality and economical point of view. Predominantly clear indication of an acuteness level (emergency: yes/no) was not provided by the requesting physician but should be part of the regularly communicated information. The particularly absence of this information makes it difficult to perform a reasonable coordination of the consultation service and to set adequate preferences. On the other hand, the arbitrary completion of the consultation letters by the consultants could decrease the treatment quality by means of missing standardization that possibly results in reduced capability of the psychiatric treatment. For those circumstances and based on these findings we conducted and established a standard form for the con- 
sulting psychiatrist that replaced the former blank documents that were the primary object of this inquiry and, as we noticed during the process of data accumulation, had been completed mostly arbitrarily. Exhibiting distinct items (basic patient data, requesting department, consultation query, anamnesis, diagnostic findings, diagnosis and therapeutic suggestions) this standard from quickly provides information to the requesting physician. Having the possibility to mark frequently neccessary statements (e.g. suidial tendency: yes/no, capacity to give informed consent: yes/no, suggested further treatment context: inpatient/outpatient) with a cross the form also reduces the psychiatrist's documentation effort. By means of this standardization the probability of occurrence of gross faults, as e.g. a missing statement towards suicidal tendency with depressive patients, can be minimized. Within the frame of acquiring more homogeneity in the outcome of our consultation psychiatry department, in a second step, a pocket-booklet regarding the most frequent mental and behavioural disorders within psychiatric consultations in our clinic as detected by our analysis was created. This pocket-booklet provided valuable information in the field of diagnostic criteria, differential diagnosis and treatment guidelines of frequently faced psychiatric comorbid disorders, and resulted in a significant relieve of consultants work, as reported in the feed back.

Organic mental disorders (e.g. dementia, delirium, including substance induced disorders) and affective disorders were the most frequently diagnosed, pointing that consultation psychiatrists should preponderantly be specialised in mentioned conditions and more over in the differential diagnostic considerations and acute intervention that makes the management of the case in an internal medicine or surgical department possible. On the other hand "no psychiatric diagnosis" was given in $12 \%$ of the cases, even though the requesting department was requiring psychiatric advice. The interpretation of this data is rather speculative, but one may postulate, that sometimes patients in somatic departments report emotional complaints that do not reach the degree of a mental disorder.

To achieve an increase of the efficiency of their PCS periodic investigations of PCS in terms of internal quality control are indispensable for every psychiatric department. Insights retrieved by those appraisals allow detecting weak points and providing basic data to create algorithms for further optimization. The necessity of quality and efficiency improvements of PCS is also reflected by the foundation of associations for consultation- and liaison-psychiatry in several countries (e.g. EACLPP: European Association of Consultation-Liaison Psychiatry and Psychosomatics, SSCLP: Association for consultation psychiatry in Switzerland, Academy of Psychosomatic Medicine: Association for consultation psychiatry in the US, Section for consultation- and liaison-psychiatry of the DGPPN: German Association for Psychiatry, Psychotherapy and Neuroscience). Those institutions offer further education and set the scientific frame for the consultation- and liaison-psychiatry in particular countries. Not least, efficiency issues implicate economic aspects as well what gives reason to account for the economic character of the relationship between the department of psychiatry as the care provider and the particular requesting department as the recipient of benefits. Thus, both sides should be interested in smooth transfer of relevant information to guarantee optimal outcome and cost-effectiveness. For that purpose we suggest an electronic request system managing the distribution of incoming consultations requests as most effective. Thereby, the requesting physician should be in charge of providing contact information to allow the psychiatrist elimination of unclarities before the consultation visit. A common problem is the evaluation of the clinical results of the psychiatric intervention in the context of PCS due to the usual single-contact setting with its difficulty to retrieve data of the patient's development after the psychiatric consultation. The electronic data system could easily been modified to allow the requesting physician to state how the patients developed and whether the psychiatric consultation was realised at all and, if so, was felt to be helpful or not.

There were some relevant limitations in our study. First, a large amount of data of the documentation was predominantly represented in metric variables and nominal scaled variables. This fact made the attribution of single cases to certain data impossible. The absence of systematically interdependence between the measured variables did not allow to target on e.g. the frequency of certain diagnoses regarding to special departments or sex or age related accents. Secondly, identification of diagnoses was based on ICD-10 diagnoses without specification of subgroups what made it impossible to distinguish between e.g. delirium and dementia in the F0 group. Least, other variables of interest as types of recommended medications, and preexisting psychiatric history have not been investigated.

\section{Conclusions}

Our retrospective analyses of the PCS displayed a useful data set to identify unfavourable idiosyncrasies of documentation on both sides of the consultation process, the psychiatrist as the care provider as well as the requesting physician, and furthermore provided basic statistical data concerning the spectrum of frequent diseases, patient 
population, frequently asked questions, etc. Lacking standardization seemed to be the main culprit in the genesis of inadequateness of our PCS. The high frequency of unspecific or even missing consultation questions judge an interdisciplinary arrangement as expedient for creating a rational algorithm for reasonable processing of the PCS. Intending rational improvement of our PCS we established standardization of our consultation reports with a standard form that was created based on our findings. In addition we created a mandatory pocketbooklet for our consultants providing useful information according to the most frequent psychiatric diseases in our clinic to generate more homogeneity in the treatment outcome. Though no prospective study concerning the outcome of these two interventions had been performed first feedbacks of our consultants were promising.

Therefore, we suggest meticulous and structured analysis of the PCS of particular hospitals as valuable for quality improvements based on the retrieved data by means of standardization. Implementation of standardized consultation sheets and a pocket-booklet with binding information together with a specific interdisciplinary arrangement to optimize communication could be elements of a possible first strategy for improvements of PCS. However, effects of interventions of that kind remain to be examined in prospective studies.

\section{References}

[1] M. Hansen, P. Fink, M. Frydenberg, M. Oxhoj, L. Sondergaard and P. Munk-Jorgensen, "Mental Disorders among Internal Medical Inpatients: Prevalence, Detection, and Treatment Status," Journal of Psychosomatic Research, Vol. 50, No. 4, 2001, pp. 199-204. doi:10.1016/S0022-3999(00)00230-0

[2] M. Martucci, M. Balestrieri, G. Bisoffi, P. Bonizzato, M. Covre, L. Cunico, et al. "Evaluating Psychiatric Morbidity in a General Hospital: A Two-phase Epidemiological Survey,” Psychological Medicine, Vol. 29, No. 4, 1999, pp. 823-832. doi:10.1017/S0033291799008491

[3] A. Seltzer, "Prevalence, Detection and Referral of Psychiatric Morbidity in General Medical Patients," Journal of the Royal Society of Medicine, Vol. 82, No. 7, 1989, pp. 410-412.

[4] P. Silverstone, "Prevalence of Psychiatric Disorders in Medical Inpatients," Journal of Nervous and Mental Disease, Vol. 184, No. 1, 1996, pp. 43-51. doi:10.1097/00005053-199601000-00008

[5] F. Lesperance, N. Frasure-Smith, M. Juneau and P. Theroux, "Depression and 1-Year Prognosis in Unstable
Angina,” Archives of Internal Medicine, Vol. 160, No. 9, 2000, pp. 1354-1360. doi:10.1001/archinte.160.9.1354

[6] S. Saravay and M. Lavin, "Psychiatric Comorbidity and Length of Stay in General Hospital. A Critical Review of Outcome Studies,” Psychosomatics, Vol. 35, No. 3, 1994, pp. 233-252.

[7] J. Wallen, H. Pincus, H. Goldman and S. Marcus, "Psychiatric Consultations in Short-term General Hospitals," Archives of General Psychiatry, Vol. 44, No. 2, 1987, pp. 163-168.

[8] L. Stevens, G. de Moore and J. Simpson, "Delirium in Hospital: Does it Increase Length of Stay?” Australian and New Zealand Journal of Psychiatry, Vol. 32, No. 6, 1998, pp. 805-808. doi:10.3109/00048679809073869

[9] A. Diefenbacher and J. Strain, "Consultation-Liaison Psychiatry: Stability and Change over a 10-Year Period," General Hospital Psychiatry, Vol. 24, No. 4, 2002, pp. 249-256. doi:10.1016/S0163-8343(02)00182-2

[10] J. Grant, W. Meller and B. Urevig, "Changes in Psychiatric Consultations over Ten Years,” General Hospital Psychiatry, Vol. 23, No. 5, 2001, pp. 261-265. doi:10.1016/S0163-8343(01)00159-1

[11] J. Su, S. Chou, C. Chang and H. Weng, "Changes in Consultation-liaison Psychiatry in the First Five Years of Operation of a Newly-Opened Hospital," Chang Gung Medical Journal, Vol. 33, No. 3, 2010, pp. 292-300.

[12] A. Diefenbacher, "A Single-Site Observational Study over a 1-Year Period," Psychosomatics, Vol. 42, No. 5, 2001, pp. 404-410. doi:10.1176/appi.psy.42.5.404

[13] J. Cooper, "Classification of Mental and Behavioural Disorders with Glossary and Diagnostic Ctiteria for Research ICD-10: DCR-10,” Churchill Livingston, Edingburgh, London, Melbourne, New York and Tokyo, 1994.

[14] H. Rothenhausler, S. Ehrentraut and H. Kapfhammer, "Changes in Patterns of Psychiatric Referral in a German General Hospital: Results of a Comparison of Two 1-Year Surveys 8 Years Apart," General Hospital Psychiatry, Vol. 23, No. 4, 2001, pp. 205-214. doi:10.1016/S0163-8343(01)00146-3

[15] Z. Lipowski and E. Wolston, "Liaison Psychiatry: Referral Patterns and Their Stability over Time," American Journal of Psychiatry, Vol. 138, 1981, pp. 249-256.

[16] A. Carson, H. Dawson, D. Marshall and K. Slatford, "Twenty-Eight Years of Liaison Psychiatry at a General Hospital,” Psychiatric Bulletin, Vol. 22, 1998, pp. 754-758. doi:10.1192/pb.22.12.754

[17] M. Hengeveld, H. Rooyamans and R. Vecht-van den Bergh, "Psychiatric Consultations in a Dutch University Hospital: A Report on 1814 Referrals, Compared with a Literature Review," General Hospital Psychiatry, Vol. 6, No. 4, 1984, pp. 271-279. doi:10.1016/0163-8343(84)90021-5 